\title{
Anesthetic considerations for an adult with Wolf- Hirschhorn syndrome \\ - A case report -
}

Received May 8, 2019

Revised 1st, May 2, 2019

2nd, June 15, 2019

Accepted June 18, 2019

\section{Corresponding author}

Hee Young Kim, M.D., Ph.D. Department of Anesthesia and Pain Medicine, Pusan National University Yangsan Hospital, 20 Geumo-ro, Mulgeum-eup, Yangsan 50612, Korea

Tel: 82-55-360-2129

Fax: 82-55-360-2149

E-mail: yuvi1981@naver.com ORCID

https://orcid.org/0000-0001-7809-8739

\section{Hye-Jin Kim 1 , Jun A You', Seyeon Park', Eun-Jung Kim², Soon Ji Park' ${ }^{1}$, and Hee Young Kim ${ }^{1}$}

${ }^{1}$ Department of Anesthesia and Pain Medicine, Pusan National University Yangsan Hospital, ${ }^{2}$ Department of Dental Anesthesia and Pain Medicine, Pusan National University Dental Hospital, Yangsan, Korea

Background: Wolf-Hirschhorn syndrome (WHS) includes features such as growth restriction, mental retardation, congenital heart disease, convulsions as well as microcephaly and micrognathia. Thus, the anesthesiologists may have difficulties in airway management, neuromuscular relaxation, and in maintaining hemodynamic stability.

Case: A 24-year-old man with WHS underwent surgery for closed reduction and internal fixation of the right neck of femur. His face showed features typical of patients with WHS such as a prominent glabella, hypertelorism, micrognathia, low-set malformed ears, and a down-turned mouth. Since difficult airway management was expected, a video-assisted laryngoscope was used for successful intubation. The surgery terminated without any problems under total intravenous anesthesia.

Conclusions: A patient with WHS may have a variety of problems. Therefore, through careful evaluation of the airway, cardiovascular system, and cerebral nervous system and by making an appropriate anesthetic plan, anesthesiologists can perform a safe general anesthesia in patients with WHS.

Keywords: Adult; Airway management; Chromosome abnormality; General anesthesia; Wolf-Hirschhorn syndrome.
Wolf-Hirschhorn syndrome (WHS) is an inherited disease caused by deletion of the short-arm (4p16.3) of chromosome 4. It has a prevalence of $1: 50,000$ and affects twice as many women as men [1]. The clinical phenotype is diverse and varies according to the amount of genetic material deleted. It includes features such as growth restriction, developmental delay, mental retardation, convulsions, and congenital heart disease (Table 1) [2].

Due to midline fusion defects, craniofacial dysmorphism is common. This, known as Greek warrior's helmet, is characterized by severe ocular hypertelorism, glabellar prominence, beaked nose, cleft lip, short philtrum, micrognathia, and microcephaly [2]. The prognosis of WHS is very poor because of lower respiratory tract infections and congenital heart diseases, with a high mortality rate of about $34 \%$ within the first two years of life [1-3]. The various deformities in WHS require various corrective surgeries under general anesthesia during childhood. The anesthesiologists may have difficulties in airway management, neuromuscular relaxation, and in maintaining hemodynamic stability. Hence, there have been several reports on anesthetic management in pediatric patients with WHS [2-7]. However, there are no case reports of anesthetic management in adults with WHS.

This is an Open Access article distributed under the terms of the Creative Commons Attribution Non-Commercial License (http://creativecommons.org/licenses/by-nc/4.0) which permits unrestricted non-commercial use, distribution, and reproduction in any medium, provided the original work is properly cited. 
Table 1. Typical Clinical Features of Wolf-Hirschhorn Syndrome and Their Incidences

\begin{tabular}{|c|c|c|c|}
\hline \multicolumn{4}{|c|}{ Incidence } \\
\hline$>75 \%$ & $50-75 \%$ & $25-50 \%$ & $<25 \%$ \\
\hline Facial features* & Skin changes & Hearing defect & Other anomalies of: \\
\hline "Greek helmet"* & Skeletal abnormality* & Cardiac defects & Liver \\
\hline Growth retardation & CF asymmetry* & Eye, optic nerve defect & Gallbladder \\
\hline Mental retardation* & Ptosis & Cleft lip palate & Gut \\
\hline Hypotonia & Abnormal teeth & GU tract defect & Diaphragm \\
\hline Lesser muscle bulk & Ab deficiency lgA & CNS defects & Esophagus \\
\hline Seizures & & Stereotypes: & Lung \\
\hline Typical EEG & & Hand washing & Aorta \\
\hline Feeding difficulties & & Hand flapping & \\
\hline & & Rocking & \\
\hline
\end{tabular}

EEG: electroencephalography, CF: craniofacial, Ab: antibody, GU: genitourinary, CNS: central nervous system. *Those marked with a asterisk are present in this patient. Reproduced from Bösenberg AT. Anaesthesia and Wolf-Hirschhorn syndrome. South Afr J Anaesth Analg 2007; 13: 31-4 [2].

\section{CASE REPORT}

We obtained the written informed consent to publication from the parent of the patient for this case report because the patient had slight mental retardation.

A 24-year-old man, with a height of $190 \mathrm{~cm}$ and weight of $52 \mathrm{~kg}$, was admitted to our hospital with right neck of femur fracture to undergo closed reduction and internal fixation. He was diagnosed with WHS after birth. Although he had slight mental retardation, he was able to ambulate and perform simple commands. No specific problems were noted on the transthoracic echocardiography, although ventricular premature beats were present on the preoperative electrocardiogram. His blood tests and chest X-ray did not show any abnormal findings.

On the physical examination, the patient had facial features identical to patients with typical WHS. The Greek warrior's helmet appearance, characterized by a prominent glabella, hypertelorism, micrognathia, low-set malformed ears, and a down-turned mouth, was present (Fig. 1A). Although mouth opening was more than 3 fingers and neck extension had no limitation, his mandible was small and relatively pushed backward (Fig. 1B). His tooth alignment and dental hygiene was under poor condition, and Mallampati score was class II. He had undergone an inguinal herniorrhaphy under general anesthesia two years prior without any complications. The information about the previous general anesthesia was limited because it was dependent on the memory from patient's mother, but any complication has not occurred during anesthetic management including endotracheal intubation.
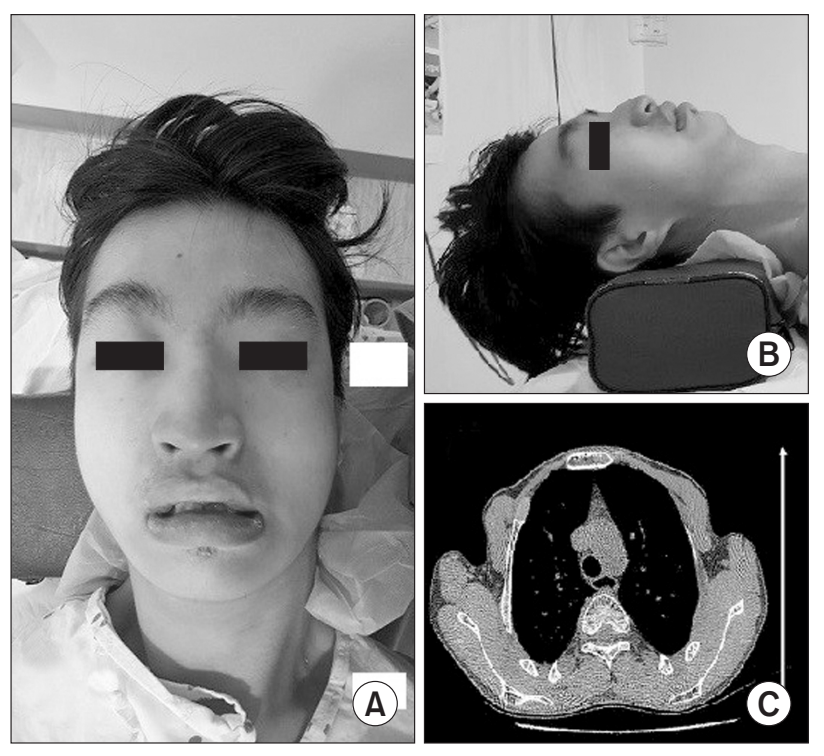

Fig. 1. Preoperative images of the adult patient with Wolf-Hirschhorn syndrome. (A, B) A prominent glabella, wide-spaced eyes, bird beakshaped nose, short philtrum, and micrognathia are seen, (C) Chest computed tomography shows the long anterior-posterior diameter of the chest cavity.

However, as a long anterior-posterior diameter of chest was noticed on computed tomography (Fig. 1C), difficult intubation and airway management were anticipated.

After entering the operating room, the hemodynamic status of the patient was stable with blood pressure of 115/91 $\mathrm{mmHg}$, heart rate of 80 beats/min, and oxygen saturation of $99 \%$. Total intravenous anesthesia was administered with $2 \%$ propofol and remifentanil to avoid malignant hyperthermia that has been previously reported during anesthesia of patients with WHS [3]. We injected $50 \mathrm{mg}$, about $1.0 \mathrm{mg} /$ 
$\mathrm{kg}$, of rocuronium intravenously while the neuromuscular transmission was monitored, considering that the usual duration of surgery is about one and a half hours or maximally two hours, the patient had no muscular hypotonia, and his mask ventilation was not challenging. As difficult intubation was anticipated, a video-assisted laryngoscope, C-MAC with D-blade (Karl Storz, Germany) was used, and endotracheal intubation with 7.5 sized reinforced endotracheal tube was delivered smoothly. The operation terminated without any problems under total intravenous anesthesia. Total duration of general anesthesia was one and a half hours and operation time was one hour. The endotracheal tube was extubated as soon as spontaneous breathing was recovered with intravenous administration of $200 \mathrm{mg}$ of sugammadex, and the ratio of train of four was confirmed to be more than $90 \%$. For reducing postoperative pain, $50 \mu \mathrm{g}$ of intravenous fentanyl was administered with patient-controlled intravenous analgesia at the end of the operation. The patient was discharged on the thirteenth postoperative day without any complications.

\section{DISCUSSION}

WHS is a rare genetic disorder with a prevalence rate of 1 : 50,000 . Although there are reports of anesthetic management in pediatric patients, knowledge of the anesthetic implications in adults with WHS is limited.

Adults with WHS are also known to have phenotypes similar to those of pediatric patients with WHS. The amount of genetic material deleted may be associated with the degree

Table 2. Anesthetic Concerns in Patients with Wolf-Hirschhorn Syndrome
1. Difficult airway management
2. Difficult vascular access
3. Associated congenital heart disease
4. Underlying seizure disorder
5. Hypotonia and choice of neuromuscular blocking agent
6. Controversial predisposition to malignant hyperthermia
7. Perioperative respiratory complications
8. Chronic aspiration
9. Non-MH-related febrile episodes (possibly secondary to chronic respiratory infections related to chronic aspiration)

MH: malignant hyperthermia. Reproduced from Humston C, Bernard R, Khan S, Tobias JD. Perioperative care of an infant with Wolf-Hirschhorn syndrome: is there a risk of malignant hyperthermia. J Med Cases 2016; 7: 126-9 [8]. of growth and developmental delay, mental retardation, seizures, and congenital cardiac dysplasia. The abnormalities in WHS, including anatomical, cardiovascular, and neuromuscular system abnormalities, may lead to various difficulties while performing general anesthesia (Table 2) [8]. Therefore, an appropriate assessment that includes preoperative history and physical examination should be performed.

The most important part of general anesthesia in patients with WHS is the difficulty of airway management in them. Smaller endotracheal tubes and airway equipment should be prepared for patients with growth retardation. The airway should be carefully evaluated before induction of general anesthesia. The anesthesiologist has to make a plan for the difficult airways in patients with craniofacial abnormalities, including micrognathia, microsomia, and web neck, since these could result in difficult laryngoscopy and intubation. If there is a risk of chronic aspiration, the anesthesiologists could consider rapid sequence intubation.

If the patient has hypotonia, the anesthesiologists should consider the type and dose of neuromuscular blocking agents (NMBA) used. Succinylcholine should be cautiously administered because it is difficult to predict how the patient will respond to it. Reducing the amount of non-depolarizing NMBA should be considered. Neuromuscular transmission should be monitored, for example the train of four while extubating, because the duration of actions of non-depolarizing NMBA, such as vecuronium, rocuronium, and cisatracurium, may be prolonged $[8,9]$.

Malignant hyperthermia (MH) after general anesthesia with inhalation anesthetics in patients with WHS has been reported [3]. However, in that report the presence of an anticholinergic crisis and active infection could not be excluded because the patient had a history of current upper respiratory infections and received high doses of atropine. In addition, according to a recent report, general anesthesia with inhalation anesthetic was safely performed in a patient with WHS without any complications $[5,6]$. Thus, anesthesiologists need to be cautious about $\mathrm{MH}$, although the association between WHS and MH seems to be poor.

WHS is usually accompanied by convulsions. Hence, anticonvulsant should be given orally, or by intravenous injection if oral administration is not possible, on the day of surgery [8]. If physical examination suggests a compromise in the cardiac function of the patient, an echocardiography should be 
performed. Prophylactic antibiotics should be administered when there are congenital cardiac defects. If the patient has mental retardation, visual impairment, or hearing impairment, it may be difficult to communicate and the patient may be uncooperative. Hence, prevention of postoperative excitement should be tried.

In summary, WHS is a very rare genetic disorder, and a patient with WHS may have a variety of problems depending on the degree of genetic defect. Therefore, through careful evaluation of the airway, cardiovascular system, and cerebral nervous system and by making an appropriate anesthetic plan, anesthesiologists can perform a safe general anesthesia in patients with WHS, as was performed in this case.

\section{CONFLICTS OF INTEREST}

No potential conflict of interest relevant to this article was reported.

\section{ORCID}

Hye-Jin Kim, https://orcid.org/0000-0003-1630-0422 Jun A You, https://orcid.org/0000-0003-1977-3671 Seyeon Park, https://orcid.org/0000-0001-7183-1811 Eun-Jung Kim, https://orcid.org/0000-0003-4982-9517 Soon Ji Park, https://orcid.org/0000-0002-3417-9972

\section{REFERENCES}

1. Paradowska-Stolarz AM. Wolf-Hirschhorn syndrome (WHS) literature review on the features of the syndrome. Adv Clin Exp Med 2014; 23: 485-9.

2. Bösenberg AT. Anaesthesia and Wolf-Hirschhorn syndrome. South Afr J Anaesth Analg 2007; 13: 31-4.

3. Ginsburg R, Purcell-Jones G. Malignant hyperthermia in the Wolf-Hirschhorn syndrome. Anaesthesia 1988; 43: 386-8.

4. Choi JH, Kim JH, Park YC, Kim WY, Lee YS. Anesthetic experience using total intra-venous anesthesia for a patient with WolfHirschhorn syndrome -a case report-. Korean J Anesthesiol 2011; 60: 119-23.

5. Mohiuddin S, Mayhew JF. Anesthesia for children with WolfHirshhorn syndrome: a report and literature review. Paediatr Anaesth 2005; 15: 254-5.

6. Iacobucci T, Nanni L, Picoco F, de Francisci G. Anesthesia for a child with Wolf-Hirshhorn syndrome. Paediatr Anaesth 2004; 14: 969.

7. Tsukamoto M, Yamanaka H, Yokoyama T. Anesthetic considerations for a pediatric patient with Wolf-Hirschhorn syndrome: a case report. J Dent Anesth Pain Med 2017; 17: 231-3.

8. Humston C, Bernard R, Khan S, Tobias JD. Perioperative care of an infant with Wolf-Hirschhorn syndrome: is there a risk of malignant hyperthermia. J Med Cases 2016; 7: 126-9.

9. Azar I. The response of patients with neuromuscular disorders to muscle relaxants: a review. Anesthesiology 1984; 61: 173-87. 